\title{
What Kind of Signaling Maintains Pluripotency and Viability in Human-Induced Pluripotent Stem Cells Cultured on Laminin-511 with Serum-Free Medium?
}

\author{
Yoshiki Nakashima* and Takeshi Omasa
}

\begin{abstract}
Xeno-free medium contains no animal-derived components, but is composed of minimal growth factors and is serum free; the medium may be supplemented with insulin, transferrin, and selenium (ITS medium). Serum-free and xeno-free culture of human-induced pluripotent stem cells (hiPSCs) uses a variety of components based on ITS medium and Dulbecco's modified Eagle's medium/Ham's nutrient mixture F12 (DMEM/F12) that contain high levels of iron salt and glucose. Culture of hiPSCs also requires scaffolding materials, such as extracellular matrix, collagen, fibronectin, laminin, proteoglycan, and vitronectin. The scaffolding component laminin-511, which is composed of $\alpha 5, \beta 1$, and $\gamma 1$ chains, binds to $\alpha 3 \beta 1, \alpha 6 \beta 1$, and $\alpha 6 \beta 4$ integrins on the cell membrane to induce activation of the PI3KJAKT- and Ras/MAPK-dependent signaling pathways. In hiPSCs, the interaction of laminin$511 / \alpha 6 \beta 1$ integrin with the cell-cell adhesion molecule E-cadherin confers protection against apoptosis through the Ras homolog gene family member A (RhoA)/Rho kinase (ROCK) signaling pathway (the major pathways for cell death) and the proto-oncogene tyrosine-protein kinase Fyn (Fyn)-RhoA-ROCK signaling pathway. The expression levels of $\alpha 6 \beta 1$ integrin and E-cadherin on cell membranes are controlled through the activation of insulin receptor/ insulin, FGF receptor/FGF2, or activin-like kinase 5 (ALK5)-dependent TGF- $\beta$ signaling. A combination of growth factors, medium constituents, cell membrane-located E-cadherin, and $\alpha 6 \beta 1$ integrin-induced signaling is required for pluripotent cell proliferation and for optimal cell survival on a laminin-511 scaffold. In this review, we discuss and explore the influence of growth factors on the cadherin and integrin signaling pathways in serum-free and xenofree cultures of hiPSCs during the preparation of products for regenerative medicinal therapies. In addition, we suggest the optimum serum-free medium components for use with laminin-511, a new scaffold for hiPSC culture.
\end{abstract}

Key words: cellular biology; cell culture; extracellular matrix; growth factor; stem cells

\section{Introduction}

Initially, human pluripotent stem cell (hPSC) lines were grown in a coculture system using a layer of mitotically inactivated mouse embryonic fibroblast (MEF) feeder cells. $^{1-3}$ However, to be clinically compliant, humaninduced pluripotent stem cells (hiPSCs) need to be cultured in a medium that is serum free, chemically defined, and free of animal products (xeno free). Serum-free medium is generally composed of minimal growth factors, bovine serum albumin (BSA) and Fraction V protein; the insulin, transferrin, and selenium (ITS) medium is supplemented with ITS. Considerable efforts are being made to develop culture systems that do not require animal feeder cells and use xeno-free medium. ${ }^{4-11}$

hPSCs cultured in MEF-conditioned medium (MEF$\mathrm{CM}$ ), which is based on ITS medium, can be maintained on Matrigel, ${ }^{9}$ a complex mixture of matrix proteins derived from Engelbreth-Holm-Swarm mouse tumors ${ }^{12-14}$; this mixture includes laminin-111, collagen IV, fibronectin, and vitronectin and supports robust hPSC growth. ${ }^{8,14-16}$ Moreover, a combination of collagen IV, fibronectin, laminin, and vitronectin can be used to

Department of Material and Life Science, Graduate School of Engineering, Osaka University, Osaka, Japan.

*Address correspondence to: Yoshiki Nakashima, DDS, PhD, Department of Material and Life Science, Graduate School of Engineering, Osaka University, U1E801, 2-1 Yamadaoka, Suita, Osaka 565-0871, Japan, E-mail: nakashimayoshiki@bio.mls.eng.osaka-u.ac.jp

() Yoshiki Nakashima and Takeshi Omasa 2016; Published by Mary Ann Liebert, Inc. This Open Access article is distributed under the terms of the Creative Commons License (http://creativecommons.org/licenses/by/4.0), which permits unrestricted use, distribution, and reproduction in any medium, provided the original work is properly credited. 
replace Matrigel for deriving and expanding hPSCs under defined culture conditions. ${ }^{8}$ A laminin-511 scaffold can bind $\alpha 3 \beta 1, \alpha 6 \beta 1$, and $\alpha 6 \beta 4$ integrins and is able to maintain hPSCs in the undifferentiated state in serum- and xeno-free medium under feeder cell-free culture conditions. ${ }^{17-21}$

In this review, we consider the effects of medium components and intracellular signal transduction in cultures using serum- and xeno-free medium and a feeder cell-free culture system. Substrates obtained from recombinant human laminin-511 offer significant improvements for establishing defined conditions for adhesion cultures of hPSCs under clinically compliant settings. We suggest that choosing the components of the culture medium and the scaffolding materials can ensure maintenance of pluripotency and cell survival through PI3K/AKT and Fyn-RhoA-ROCK signaling pathways in hiPSC culture.

Signaling Pathways in Serum-Free Culture Systems hiPSCs easily lose pluripotency due to a component in serum. Therefore, hiPSCs have to be cultured using serum-free culture medium. Initially, researchers examined serum albumin, a primary component of serum, and found that Dulbecco's modified Eagle's medium/ Ham's nutrient mixture F12 (DMEM/F12) culture medium supplemented with serum albumin, FGF2, and the ITS medium components. ITS functioned similarly as serum-based culture media for hiPSCs. However, the serum-free medium used for the culture of hiPSCs initially contained a component derived from an animal. In addition, mouse feeder cells were also used.

\section{EFGF2/FGFR signaling}

FGFR1 (fibroblast growth factor receptor 1) is the predominant FGFR in hiPSCs. Binding of FGF2 activates FGFR and major signal transduction pathways, such as all three branches of the PI3K and AKT pathways, the mitogen-activated protein kinase (MAPK) pathway, and the extracellular signal-regulated kinase (ERK)/ MAPK pathway. FGF2 activates the MAP kinases ERK1/ERK2, and also PI3K/AKT and c-Jun, and stimulates signaling cascades by activation of activin $\mathrm{A}^{22,23}$ Induction of $\alpha 2 \beta 1, \alpha 3 \beta 1, \alpha 6 \beta 1$, and $\alpha 6 \beta 4$ integrin transcription requires the FGF2-FGFR signal pathway and is reinforced by the ALK4-activin A signaling pathway. FGF2 signaling may act through the PI3K pathway to regulate the synthesis of pericellular laminin-332, -511, -521 , and collagen IV isotypes and provide a framework for the basement membrane of hPSCs. ${ }^{24}$ Blocking the
FGF2 pathway in hPSCs can lead to differentiation., ${ }^{6,8,25}$ Extracellular matrix (ECM) modulated signals may converge with FGF2 signaling and be involved in the maintenance of pluripotency (Fig. 1).

Bovine serum albumin

BSA has many roles in cell culture; it acts as a lipid carrier, provides physical protection, ${ }^{26,27}$ and prevents toxic effects by $\beta$-mercaptoethanol (a reducing agent normally required for culturing cells). In addition, BSA acts as a nutrient for MEFs. Many proteins secreted by MEFs have been identified recently in mass spectrometric analyses. ${ }^{28-30}$ MEF secreted heparan sulfate proteoglycans (HSPG), fibronectin, transforming growth factor $\beta$ (TGF- $\beta$ ), and insulin-like growth factors 1 (IGF1) and 2 (IGF2). In addition, it has been shown that critical products produced by MEF feeder layers include activin $\mathrm{A}^{31}$ (Fig. 1).

\section{Insulin/IGF-R signaling}

The IGF-1R signaling axis is critical to cell proliferation and glucose metabolism and also induces E-cadherin transcription in hiPSCs. Upon binding insulin, IGF-1R is activated through autophosphorylation. PI3K activation then leads to an increase in phosphatidylinositol 3,4,5trisphosphate (PIP3), which results in the activation of the critical protein AKT. ${ }^{25}$ This signaling axis is commonly referred to as the PI3K/AKT, nuclear factor $\kappa \mathrm{B}$ $(\mathrm{NF}-\kappa \mathrm{B}$ ), and jun $\mathrm{N}$-terminal kinases (JNK) pathway and as c-Jun phosphorylation of IGF-1R signaling; it is ultimately responsible for the maintenance of hPSCs and hiPSCs. ${ }^{32,33}$ In parallel, IGF-1R signaling also promotes cell proliferation through the Ras/MAPK pathway (Fig. 1).

\section{Transferrin}

Transferrin, a glycoprotein, is required in the culture medium to act primarily as an iron transporter and secondarily to bind endogenous iron. Transferrin enables the cells to take up oxygen and also stimulates growthrelated enzyme activities. Two types of transferrin are generally added to culture media: iron-saturated transferrin (holo-transferrin) and iron-deficient transferrin (apo-transferrin) (Fig. 1).

\section{Selenium}

Selenium, in the form of selenoproteins in particular glutathione peroxidase (Glu.Px), is an essential component of the human cellular antioxidant defense system. It also has anti-inflammatory effects by its ability to scavenge free radicals. ${ }^{34}$ As is evident from Figure 1, selenium 


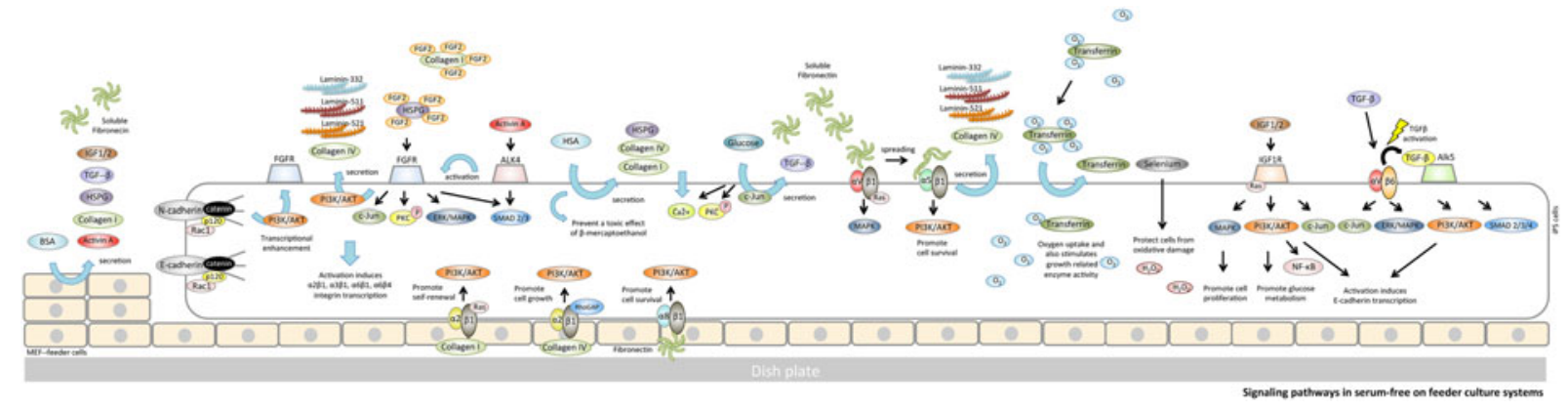

FIG. 1. Signaling pathways in serum-free on feeder culture systems. BSA acts as a nutrient for MEFs. MEFs secrete heparan sulfate proteoglycans (HSPG), fibronectin, TGF- $\beta$, IGF1, and IGF2. HSA acts as a nutrient for hiPSCs. Albumin in the culture medium promotes expression of collagen I, collagen IV, and HSPG that detoxify $\beta$ mercaptoethanol. Collagen I binds to $\alpha 2 \beta 1$ integrin and transduces Ras function to activate PI3K binding to AKT; activation of the latter pathway promotes self-renewal in hiPSCs. Collagen IV binds to $\alpha 2 \beta 1$ integrin and transduces a RhoGAP function to activate PI3K binding to AKT; activation of the latter pathway promotes cell growth in hiPSCs. With regard to downstream functions in the IGF1R and ALK5 pathways of hiPSCs, activation induces E-cadherin transcription that recruits the PI3K/AKT and ERK/MAPK. This suggests that both PI3K/AKT and MAPK/ERK signaling are required for insulin- and TGF- $\beta$-induced E-cadherin upregulation on the cell membrane. Glucose promotes fibronectin and TGF- $\beta$ manifestation through c-Jun signaling. TGF- $\beta$ is activated by $\alpha \mathrm{V} \beta 6$ integrin. The pathways that IGF1R and ALK5 activate through unidentified growth factors, which are present in the culture medium for hiPSCs, are sufficient to maintain pluripotency in hiPSCs. Transferrin regulates cellular iron uptake and, thus, is an essential culture medium ingredient. Selenium is a component of some glutathione peroxidases; these enzymes prevent oxidative damage in cells by inducing the reduction of lipid hydroperoxides. With regard to downstream functions in the FGFR and ALK4 pathways of hiPSCs, the induction of $\alpha 2 \beta 1, \alpha 3 \beta 1$, $\alpha 6 \beta 1$, and $\alpha 6 \beta 4$ integrin transcription requires the FGF2-FGFR signal pathway and is reinforced by ALK4-activin A signal pathway. Collagen I and HSPG concentrate on FGF2 in the vicinity of FGFR to activate laminin-332, -511, 521 , and collagen IV transcription through the PI3K/AKT signaling pathway. Fibronectin is secreted by hiPSCs and binds with $\alpha 8 \beta 1$ integrin to promote cell survival of hiPSCs through PI3K/AKT signaling. In addition, fibronectin can also bind to $\alpha \mathrm{V} \beta 1$ integrin and $\alpha 5 \beta 1$ integrin to promote secretion of laminin-332, $-511,-521$, and collagen IV from hiPSCs. The pathways activated by fibronectin-integrin signaling are responsible for the secretion of ECM, which maintains pluripotency of hiPSCs. ALK4, activin-like kinase receptor-4; BSA, bovine serum albumin; ECM, extracellular matrix; ERK, extracellular signal-regulated kinase; hiPSCs, human-induced pluripotent stem cells; FGFR, fibroblast growth factor receptor; HSA, human serum albumin; IGF, insulin-like growth factor; IGF1R, insulinlike growth factor-1 receptor; MAPK, mitogen-activated protein kinase; MEF, mouse embryonic fibroblast; PI3K, phosphatidylinositol 3-kinase; TGF- $\beta$, transforming growth factor $\beta$.

is a component of some glutathione peroxidases; these enzymes prevent oxidative damage in cells by inducing the reduction of lipid hydroperoxides.

\section{Glucose}

In hPSCs, the c-jun N-terminal kinase (JNK) inhibitor SP600125 blocks fibronectin expression induced by high glucose and inhibits cell cycle regulatory protein expression induced by high glucose-mediated TGF- $\beta$ expression. ${ }^{35}$ High glucose levels $(4.5 \mathrm{~g} / \mathrm{L})$ also increase intracellular calcium $\left(\mathrm{Ca}^{2+}\right)$ concentration, PKC phosphorylation, and TGF- $\beta$ expression in hPSCs. Thus, c-Jun is necessary for expression of fibronectin and TGF- $\beta$ (Fig. 1).

\section{Signaling Pathways in Feeder Cell-Free Culture Systems}

When hiPSCs are cultured on an MEF feeder layer, the MEFs provide proteins, such as activin A, TGF- $\beta$, IGF1 and 2 (IGF2), and glycans, including HSPG, fibronectin, 
collagen I, collagen IV, and laminin. These proteins and glycans are necessary for hiPSC attachment, proliferation, and self-renewal ${ }^{36}$ (Fig. 1). Matrigel, which includes laminin-111, collagen IV, fibronectin, and vitronectin, can be used to replace the MEF feeder layer and support robust hPSC growth. ${ }^{12-14}$

\section{Activin A/ALK4 signaling}

Activin A has a role in a wide range of cellular processes from cell proliferation and differentiation to apoptosis. Initially, activin A binds to type II activin A receptors (ActRIIA or ActRIIB) and then recruits type IB activin A receptor (ALK4). ALK4 interacts with and phosphorylates the SMAD family members 2 and 3 (SMAD2 and SMAD3). Vallier et al. ${ }^{37-39}$ demonstrated that the cooperation of the activin A pathway and FGF2 is necessary for the maintenance of pluripotency in hPSCs. ${ }^{40}$ When active, PI3K/AKT establishes conditions where activin A/SMAD2/3 stimulate self-renewal in hiPSCs by activating target genes, including Nanog. ${ }^{41,42}$ This stimulatory effect involves the interaction of activin A and SMAD2/3, and may also require upregulation of FGF2 pathways ${ }^{39,43}$ (Fig. 2).

\section{TGF- $\beta /$ ALK5 signaling}

TGF- $\beta$ type 1 receptor, also known as ALK5, phosphorylates SMAD2/3 and forms a complex with SMAD4 that translocates to the nucleus. The complex induces transcription of TGF- $\beta$-dependent genes that regulate signaling pathways involved in pluripotency and self-renewal, such as the MAPK and PI3K/ AKT pathways. Signaling through these pathways supports proliferation and continued pluripotency in hiPSCs ${ }^{44}$ (Fig. 2).

\section{Signaling Pathways in Xeno-Free Culture Systems}

Animal serum contains xenogeneic antigens, and cells cultured in serum have the potential to induce immune reactions in recipients and also raise the risk of transmission of animal viruses, prions, and zoonotic infections. Thus, animal serum is not desirable for clinical cell production or for transplantation therapies. Laminin-511 scaffold can bind $\alpha 3 \beta 1, \alpha 6 \beta 1$, and $\alpha 6 \beta 4$ integrins and can be used to maintain hPSCs in the undifferentiated state under serum and xeno-free conditions in a feeder cell-free culture system. ${ }^{17-21}$

\section{Recombinant human albumin}

Human albumin has many biological and physical roles in hiPSC culture and is necessary for StemFit medium (Ajinomoto Co., Inc.) to enable the efficient formation of colonies by hiPSCs on recombinant laminin-511. ${ }^{45}$ However, albumin in the culture medium promotes the secretion of ECM molecules such as collagen I, collagen IV, and HSPG. Consequently, protein lytic enzymes such as the trypsin

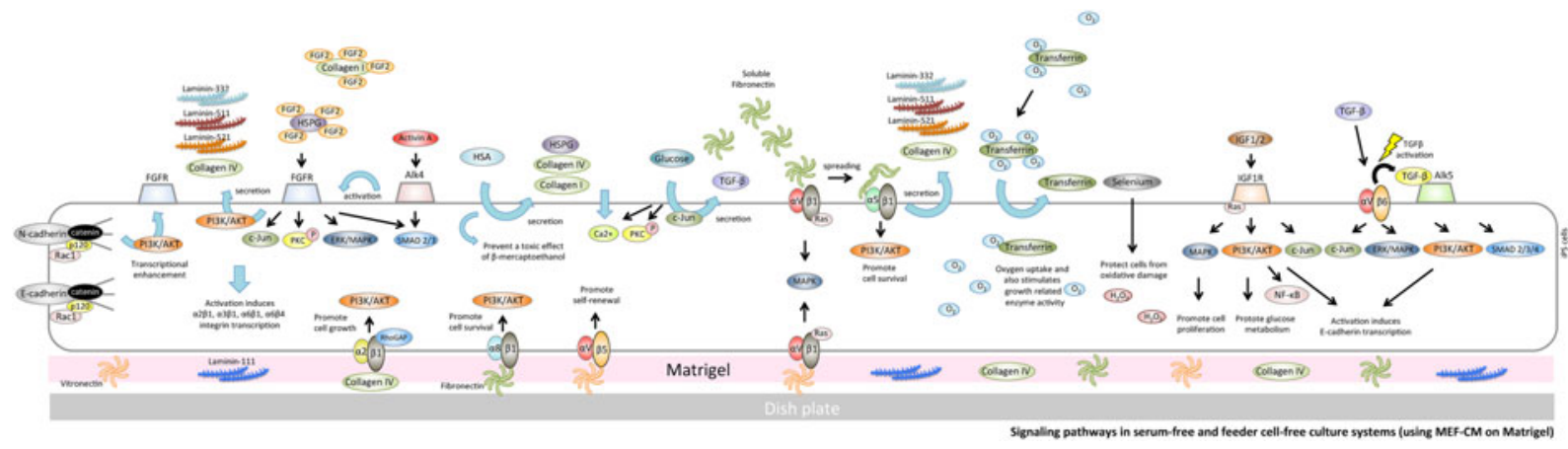

FIG. 2. Signaling pathways in serum-free and feeder cell-free culture systems (using MEF-CM on Matrigel). Matrigel includes laminin-111, collagen IV, fibronectin, and vitronectin and supports robust hPSC growth. Collagen IV binds to $\alpha 2 \beta 1$ integrin and transduces a RhoGAP function to activate PI3K binding to AKT; activation of the latter pathway promotes cell growth in hiPSCs. Vitronectin supports the maintenance of hPSCs through $\alpha \mathrm{V} \beta 5$ integrin. $\alpha \mathrm{V} \beta 1$ integrin is able to recognize vitronectin and fibrinogen. Fibronectin can also bind to $\alpha \mathrm{V} \beta 1$ integrin and $\alpha 5 \beta 1$ integrin to promote secretion of laminin-332, $-511,-521$, and collagen IV from hiPSCs. hPSC, human pluripotent stem cells; MEF-CM, MEF-conditioned medium. 
are necessary to break the strong cell-to-plate adhesion (Fig. 3).

\section{Recombinant vitronectin scaffold}

Recombinant vitronectin as a glycoprotein can function as a chemically defined matrix component in hiPSCs. Vitronectin is a functionally defined substrate that supports hPSCs self-renewal through $\alpha \mathrm{V} \beta 5$ integrin in albuminfree medium such as Essential 8 medium (Thermo Fisher Scientific). It is possible to dislodge cells from the culture plate using only EDTA; thus, integrins on the cell surface are not damaged by trypsin treatment.

\section{Recombinant laminin-511 scaffold}

The scaffolding component laminin-511, which is composed of $\alpha 5, \beta 1$, and $\gamma 1$ chains, binds to $\alpha 3 \beta 1, \alpha 6 \beta 1$, and $\alpha 6 \beta 4$ integrins on the cell membrane to induce activation of the PI3K/AKT- and Ras/MAPK-dependent signaling pathways. In hiPSCs, the interaction of laminin-511/ $\alpha 6 \beta 1$ integrin with the cell-cell adhesion molecule E-cadherin confers protection against apoptosis through the Ras homolog gene family member A (RhoA)/Rho kinase (ROCK) signaling pathway (the major pathway for cell death) and the proto-oncogene tyrosine-protein kinase Fyn (Fyn)-RhoA-ROCK signaling pathway.

\section{Signaling Pathways in Cultures Using Laminin-511 Cadherin}

Cell adhesion molecules (CAMs), such as cadherins and integrins, stimulate intercell adhesion. The "classical" CAMs include epithelial (E-), neural (N-), and placental (P-) cadherins and, as their names suggest, they tend to be expressed in a tissue-specific pattern. ${ }^{46-52}$ In addition to their role in cell-cell adhesion, cadherins may also function in signaling pathways between the cell membrane and the nucleus. ${ }^{53}$

E-cadherin signaling. E-cadherin, a $\mathrm{Ca}^{2+}$-dependent cell-cell adhesion molecule, ${ }^{50,54}$ is stabilized at the cell surface by its link to the actin cytoskeleton through $\beta$-catenin and $\alpha$-catenin and is essential for PSC intercellular adhesion and colony formation. ${ }^{55,56}$ It has been shown that functional interaction between Rac1, a small Rho family GTPase, and E-cadherin is responsible for regulating hPSC self-renewal. ${ }^{57,58}$ E-cadherin-mediated adhesion is commonly associated with $\beta$-catenin signaling and also stimulates PI3K/AKT signaling. ${ }^{59,60}$ The ability of the PI3K/AKT pathway to promote cell survival indicates a broader role for Rho family GTPases through p120-catenin. ${ }^{61,62}$ The PI3K inhibitor wortmannin prevents E-cadherin-dependent Racl activation, but does not interfere with the intracellular distribution of Racl

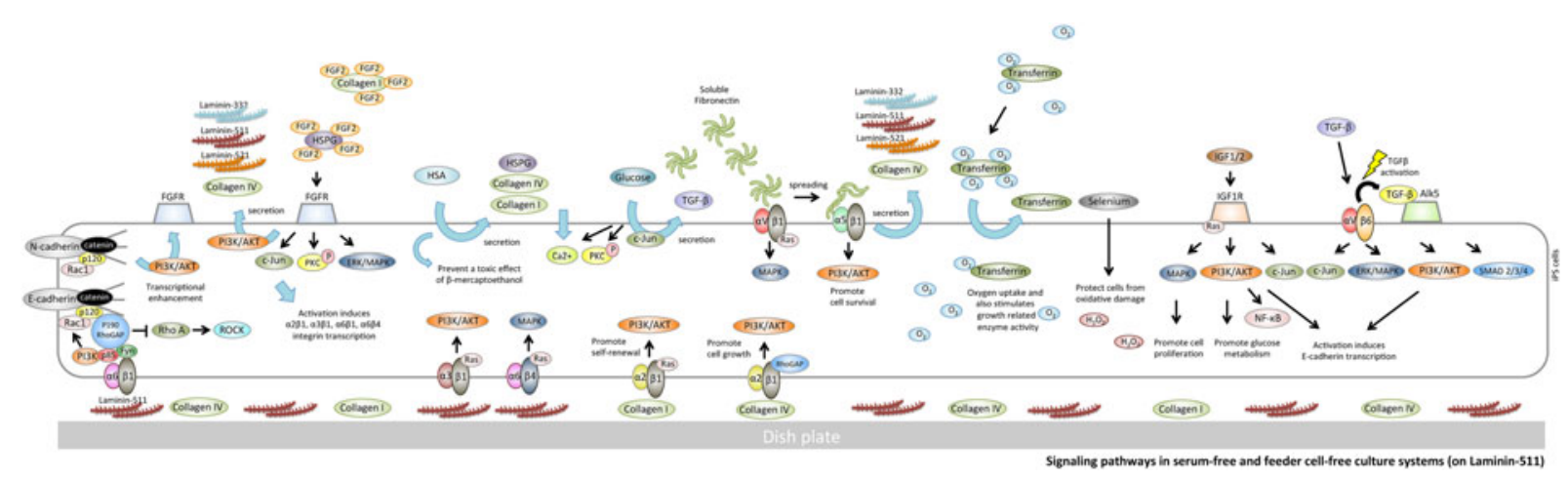

FIG. 3. Signaling pathways in serum-free and feeder cell-free culture systems (on laminin-511). Cadherin is activated by a cell junction. When $\mathrm{N}$-cadherin is activated, transcriptional enhancement of FGFR occurs. As a result, the FGF2 signal pathway is activated. This signaling pathway has an important role in the expression of FGFR attachment to the cell membrane. In cultures using a laminin-511 scaffold, the E-cadherin- $\alpha 6 \beta 1$ integrin signal pathway is required to control cell death in hiPSCs. PI3K signaling promotes expression of Rac1. Rac1 binds to cadherins, and cadherins prevent endocytosis. When $\alpha 6 \beta 1$ integrin is combined with laminin-511, FynRhoA-ROCK signaling is induced. As a result, hiPSC death caused by ROCK is repressed. Laminin-511 can directly bind to $\alpha 3 \beta 1$ integrin and, thereby, activate the PI3K/AKT signaling pathway through Ras. In addition, linkage of $\alpha 6 \beta 4$ activates the MAP kinase pathways directly through Ras. 
or E-cadherin. ${ }^{63}$ The activation of Racl through PI3K by E-cadherin-mediated cell-cell adhesion involves at least two steps as follows: (1) recruitment of Racl to E-cadherin mediates cell-cell contact sites and (2) Rac1 activation by a guanine nucleotide exchange factor. ${ }^{64}$ E-cadherin levels at adherens junctions are regulated by endocytosis and recycling; activated Racl reduces E-cadherin endocytosis and, thus, increases the levels of cell surface E-cadherin and, consequently, promotes cell-cell adhesion. Ectopic overexpression of E-cadherin also increases the rate of survival in dissociated hPSCs. ${ }^{65}$ However, when dissociated hPSCs are grown on Ecadherin-coated plates, they form membrane protrusions and have a lower rate of survival. Thus, E-cadherin interactions alone are not the only factor in cell survival. ${ }^{66}$ Transcription factors for E-cadherin expression appear to act downstream in various signaling pathways, such as in the TGF- $\beta$, FGF2, NF- $\kappa$ B, and integrin cascades ${ }^{67}$ (Fig. 3).

$\mathrm{N}$-cadherin signaling. N-cadherin induces FGFR upregulation $^{68}$ and has also been shown to stimulate FGF2 signaling. ${ }^{69} \mathrm{~N}$-cadherin adhesion can lead to PI3K-mediated activation of $\mathrm{AKT},{ }^{70,71}$ and activated AKT signaling can stimulate $\beta$-catenin signaling. The E-cadherin function can be replaced by N-cadherin in the rescue of the undifferentiated pluripotent iPSC state ${ }^{72}$ (Fig. 3).

P-cadherin signaling. P-cadherin controls cell-tolaminin adhesion by modulating the expression and activation of the laminin receptor $\alpha 6 \beta 4$ integrin heterodimer. ${ }^{73}$ Importantly, it has been reported that $\alpha 6 \beta 4$ integrin (adhering to laminin-332-containing $\alpha 3, \beta 3$, and $\gamma 2$ chains) promotes survival and invasion by activating the PI3K/AKT pathway. ${ }^{74-76} \alpha 6$ Integrin activation induces $\mathrm{P}$-cadherin transcription. ${ }^{77} \mathrm{P}$-cadherin results in the translocation of p120ctn from the plasma membrane to the cytoplasm, thereby activating Rac1. ${ }^{78}$

\section{Integrin}

Integrins are transmembrane proteins that have a wide range of functions, including adhesion and cell-cell interactions. ${ }^{79}$ They are heterodimeric molecules consisting of $\alpha$ and $\beta$ chains. The 24 different heterodimeric combinations of integrin subunits in vertebrates have different ligand binding preferences. Based on their ligand specificities, the integrin family can be divided into three subgroups with respect to hPSC culture: collagen receptors $(\alpha 2 \beta 1$ and $\alpha 11 \beta 1)$, laminin receptors $(\alpha 3 \beta 1, \alpha 6 \beta 1, \alpha 7 \beta 1$, and $\alpha 6 \beta 4$ ), and RGD (Arg-Gly-
Asp) receptors $(\alpha 5 \beta 1, \alpha 8 \beta 1, \alpha \mathrm{V} \beta 1, \alpha \mathrm{V} \beta 5, \alpha \mathrm{V} \beta 6$, and $\alpha \operatorname{IIb} \beta 3){ }^{36,80-82}$

\section{Collagen receptors $(\alpha 2 \beta 1$ and $\alpha 11 \beta 1)$}

Total collagen complex, including types I, II, III, and IV, can induce an increased proliferative capacity in iPSCs. ${ }^{83}$ The effect of collagen I and IV on cell migration is mediated by $\alpha 2 \beta 1$ integrin. ${ }^{84,85}$ Collagen is not included in cultures using a laminin-511 scaffold, although hiPSC lines do express both collagen I and IV. ${ }^{86,87}$ Albumin in the culture medium promotes collagen secretion by hiPSCs. Collagen I has been reported to stabilize FGF2, similar to HSPG, for floating cultures of hiPSCs. ${ }^{88}$

$\alpha 2 \beta 1$ integrin/collagen I/IV signaling. Collagen Ibound $\alpha 2 \beta 1$ integrin stimulates the self-renewal of PSCs by increasing the PI3K/AKT signal through the GTP Ras pathway. ${ }^{89}$ Collagen IV pretreatment enhances in vivo growth of undifferentiated PSCs without affecting their pluripotency through $\alpha 2 \beta 1$ integrin-mediated actin remodeling and the regulatory molecules PI3K and Rhofamily GTPases ${ }^{84}$ (Fig. 3).

Laminin receptors $(\alpha 3 \beta 1, \alpha 6 \beta 1, \alpha 7 \beta 1$, and $\alpha 6 \beta 4)$

Laminins are large glycoprotein molecules consisting of $\alpha, \beta$, and $\gamma$ chains. To date, five $\alpha$, three $\beta$, and three $\gamma$ chains have been identified. Laminin expression is present in early embryos and in pluripotent mouse and human ESCs. Cultured pluripotent hPSCs express a laminin-511 heterotrimer ( $\alpha 5 \beta 1 \gamma 1)$, consisting of $\alpha 5$, $\beta 1$, and $\gamma 1$ laminin chains. ${ }^{90}$

$\alpha 3 \beta 1$ integrin/laminin-332, $-511,-521$ signaling. The role of integrin $\alpha 3 \beta 1$ in cell migration is unclear. Laminin-332 drives PI3K activation and cell survival. ${ }^{76}$ Gu et al. ${ }^{91}$ demonstrated that laminin-511 and laminin521 directly bind to $\alpha 3 \beta 1$ integrin and, thereby, activate the PI3K/AKT signaling pathway (Fig. 3). However, it is not yet clear whether laminin-511 and laminin-332 use the same or different binding sites in these receptors.

$\alpha 6 \beta 1$ integrin/laminin-332,-511,-521 signaling. Laminin511 and laminin-521 maintain hPSC pluripotency after $\alpha 6 \beta 1$ integrin signaling to induce the PI3K/AKT signaling pathway. ${ }^{90,92}$ In cultures using a laminin-511 scaffold, the interaction between laminin-511 and $\alpha 6 \beta 1$ integrin partly compensates for the ROCK inhibitor, Y-27632. The protooncogene tyrosine-protein kinase $\mathrm{Fyn}^{93}$ associates with the p85 subunit of PI3K. The Fyn-RhoA-ROCK signaling 
cascade $^{94}$ is of interest since Fyn directly associates with $\alpha 6 \beta 1$-integrin. ${ }^{95,96}$ PSCs interact with laminin-511 through $\beta 1$-integrins, mostly $\alpha 6 \beta 1$. Strong Fyn-RhoA-ROCK signaling is expected in cultures using laminin-511 as the scaffold (Fig. 3).

$\alpha 6 \beta 4$ integrin/laminin-332, $-511,-521$ signaling. Upon binding to laminin-332, the integrin $\alpha 6 \beta 4$ can organize hemidesmosomes, which are essential for stable cell adhesion. ${ }^{97,98}$ Furthermore, FGF2 upregulates the expression of $\alpha 2 \beta 1, \alpha 3 \beta 1, \alpha 6 \beta 1$, and $\alpha 6 \beta 4$ integrins in cells cultured in vitro. ${ }^{99}$ Therefore, it is believed that $\alpha 6 \beta 4$ integrin in hiPSC membranes increases if concentrated FGF2 is added to the culture medium (Fig. 3).

RGD receptors ( $\alpha 5 \beta 1, \alpha 8 \beta 1, \alpha \mathrm{V} \beta 1, \alpha \mathrm{V} \beta 5$, and $\alpha \mathrm{V} \beta 6)$ The tripeptide sequence arginine-glycine-aspartic acid (RGD) has been suggested to be a recognition sequence for integrins. ${ }^{100}$ This peptide sequence has been identified in many ECM proteins such as fibronectin, vitronectin, fibrinogen, von Willebrand factor, thrombospondin, laminin, entactin, tenascin, osteopontin, and bone sialoprotein. ${ }^{101,102}$

$\alpha 5 \beta 1$ integrin/fibronectin signaling. Human fibronectin, which binds to integrin $\alpha 5 \beta 1,{ }^{103}$ supports hPSC expansion in serum-free medium. ${ }^{104} \alpha 5 \beta 1$ integrin binds soluble fibronectin efficiently; first, the $\alpha 5$ integrin binds to the synergy site to cause a conformational change in the fibronectin; second, $\beta 1$ integrin binds to the exposed RGD sequence. ${ }^{105}$ Overexpression of $\alpha 5 \beta 1$ provides considerable protection against several proapoptotic stimuli. Furthermore, the protective effects of $\alpha 5 \beta 1$ are mediated through a PI3K/AKTdependent pathway ${ }^{106}$ (Fig. 3).

$\alpha 8 \beta 1$ integrin/fibronectin signaling. $\alpha 8 \beta 1$ integrin, a cell adhesion receptor that links the cytoskeleton to ECM proteins, not only mediates mechanical and chemical signals from the matrix but also regulates cell adhesion through bidirectional membrane transduction. ${ }^{107}$ Under serum deprivation conditions, $\alpha 8 \beta 1$ can interact with fibronectin to increase cell survival rates so that $\alpha 8$-mediated survival is mediated by the PI3K pathway ${ }^{108}$ (Fig. 2).

$\alpha \mathrm{V} \beta 1$ integrin/fibronectin signaling. $\alpha \mathrm{V} \beta 1$ has been described as a receptor for fibronectin, ${ }^{109}$ vitronectin, ${ }^{110,111}$ or collagen. ${ }^{112} \alpha \mathrm{V} \beta 1$ integrin is able to recognize vitronectin and fibrinogen and also cooperate with $\alpha 5 \beta 1$ to mediate attachment to and spreading on fibronectin; ${ }^{91,113}$ Ras/MEK/MAPK signaling and kinases are also stimulated by integrin ligation (Fig. 2).

$\alpha \mathrm{V} \beta 5$ integrin/vitronectin signaling. Recombinant vitronectin is a functionally defined substrate that supports hPSCs self-renewal through $\alpha \mathrm{V} \beta 5$ integrin ${ }^{36}$ (Fig. 4). $\alpha \mathrm{V} \beta 5$ activates latent TGF- $\beta .^{114}$ Chen et al.

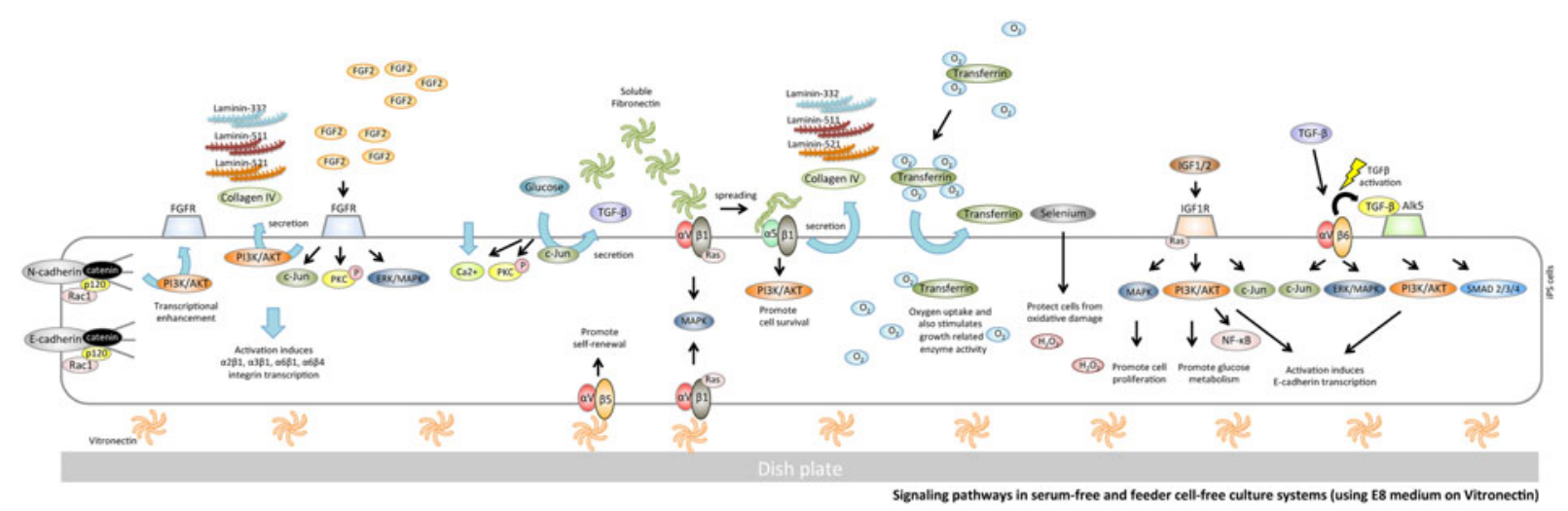

FIG. 4. Signaling pathways in serum-free and feeder cell-free culture systems (using E8 medium on vitronectin). Recombinant vitronectin is a functionally defined substrate that supports hPSCs self-renewal through $\alpha \mathrm{V} \beta 5$ integrin. $\alpha \mathrm{V} \beta 1$ has been described as a receptor for vitronectin through Ras/MEK/MAPK signaling, and kinases are also stimulated by integrin ligation. This culture system does not need albumin; as collagen I and HSPG do not concentrate on FGF2, it is necessary to have a high concentration of FGF2 in the culture medium. 
enhanced the attachment and survival of hPSCs on vitronectin-coated surfaces by excising the $\mathrm{N}$-terminal and/or C-terminal fragments of full-length vitronectin. ${ }^{32}$ It has also been reported that vitronectin and one of its variants (VTN-NC) support the maintenance of hPSCs through $\alpha \mathrm{V} \beta 5$ integrin. ${ }^{32,36,115}$

$\alpha \mathrm{V} \beta 6$ integrin/TGF- $\beta$ signaling. $\alpha \mathrm{V} \beta 6$ integrin has a key role in regulating TGF- $\beta$ activation of ALK5, a TGF- $\beta$ type I receptor. ${ }^{114,116,117}$ The association with $\alpha \mathrm{V} \beta 6$ integrin expression may be linked to TGF- $\beta$ activation, which has an important role in intracellular signaling. TGF- $\beta$ activates c-Jun and Erk through $\alpha \mathrm{V} \beta 6$ integrin. TGF- $\beta$ is an essential additive in culture medium using a laminin-511 scaffold (Fig. 3).

\section{Conclusion}

This review highlights the components that are suitable for serum- and xeno-free media and feeder cell-free culture systems that use laminin-511 as the scaffold. The binding of $\alpha 6 \beta 1$ with laminin-511 and the interaction with E-cadherin stimulate Fyn-RhoA-ROCK signaling to act as a deactivation ROCK signal. These results indicate that the signaling pathways induced by a laminin-511 scaffold depend on PI3K signaling through E-cadherin-mediated cell-cell adhesion and that the Fyn-RhoA-ROCK signaling cascade is associated with $\alpha 6 \beta 1$ integrin-laminin-511 adhesion.

\section{Author Disclosure Statement}

No competing financial interests exist.

\section{References}

1. Reubinoff $B E$, Pera MF, Fong $C Y$, et al. Embryonic stem cell lines from human blastocysts: somatic differentiation in vitro. Nat Biotechnol. 2000;18:399-404.

2. Takahashi K, Tanabe K, Ohnuki M, et al. Induction of pluripotent stem cells from adult human fibroblasts by defined factors. Cell. 2007;131:861-872.

3. Thomson JA, Itskovitz-Eldor J, Shapiro SS, et al. Embryonic stem cell lines derived from human blastocysts. Science. 1998;282:1145-1147.

4. Beattie GM, Lopez AD, Bucay N, et al. Activin A maintains pluripotency of human embryonic stem cells in the absence of feeder layers. Stem Cells. 2005;23:489-495

5. Klimanskaya I, Chung Y, Meisner L, et al. Human embryonic stem cells derived without feeder cells. Lancet. 2005;365:1636-1641.

6. Levenstein ME, Ludwig TE, Xu RH, et al. Basic fibroblast growth factor support of human embryonic stem cell self-renewal. Stem Cells. 2006;24:568-574.

7. Li Y, Powell S, Brunette E, et al. Expansion of human embryonic stem cells in defined serum-free medium devoid of animal-derived products. Biotechnol Bioeng. 2005;91:688-698.

8. Ludwig TE, Levenstein ME, Jones JM, et al. Derivation of human embryonic stem cells in defined conditions. Nat Biotechnol. 2006;24:185187.

9. Xu C, Inokuma MS, Denham J, et al. Feeder-free growth of undifferentiated human embryonic stem cells. Nat Biotechnol. 2001;19:971-974.
10. Xu C, Rosler E, Jiang J, et al. Basic fibroblast growth factor supports undifferentiated human embryonic stem cell growth without conditioned medium. Stem Cells. 2005;23:315-323.

11. Xu RH, Peck RM, Li DS, et al. Basic FGF and suppression of BMP signaling sustain undifferentiated proliferation of human ES cells. Nat Meth. 2005;2:185-190.

12. Stover $\mathrm{AE}$, Schwartz $\mathrm{PH}$. Adaptation of human pluripotent stem cells to feeder-free conditions in chemically defined medium with enzymatic single-cell passaging. Methods Mol Biol. 2011;767:137-146.

13. Totonchi M, Taei A, Seifinejad A, et al. Feeder- and serum-free establishment and expansion of human induced pluripotent stem cells. Int J Dev Biol. 2010;54:877-886.

14. Kleinman HK, McGarvey ML, Liotta LA, et al. Isolation and characterization of type IV procollagen, laminin, and heparan sulfate proteoglycan from the EHS sarcoma. Biochemistry. 1982;21:6188-6193.

15. Bissell DM, Arenson DM, Maher JJ, et al. Support of cultured hepatocytes by a laminin-rich gel. Evidence for a functionally significant subendothelial matrix in normal rat liver. J Clin Invest. 1987;79:801-812.

16. Horejs CM, Serio A, Purvis A, et al. Biologically-active laminin-111 fragment that modulates the epithelial-to-mesenchymal transition in embryonic stem cells. Proc Natl Acad Sci USA. 2014;111:5908-5913.

17. Gao J, DeRouen MC, Chen $\mathrm{CH}$, et al. Laminin-511 is an epithelial message promoting dermal papilla development and function during early hair morphogenesis. Gene Dev. 2008;22:2111-2124.

18. Hakala H, Rajala K, Ojala M, et al. Comparison of biomaterials and extracellular matrices as a culture platform for multiple, independently derived human embryonic stem cell lines. Tissue Eng Part A. 2009;15:1775-1785.

19. International Stem Cell Initiative Consortium, Akopian V, Andrews PW, et al. Comparison of defined culture systems for feeder cell free propagation of human embryonic stem cells. In Vitro Cell Dev Biol Anim. 2010;46:247-258

20. Chin AC, Padmanabhan J, Oh SK, et al. Defined and serum-free media support undifferentiated human embryonic stem cell growth. Stem Cells Dev. 2010;19:753-761.

21. Rajala K, Hakala H, Panula S, et al. Testing of nine different xeno-free culture media for human embryonic stem cell cultures. Hum Reprod. 2007;22:1231-1238.

22. Feng GS. Shp2-mediated molecular signaling in control of embryonic stem cell self-renewal and differentiation. Cell Res. 2007;17:37-41.

23. Saxena S, Hanwate M, Deb K, et al. FGF2 secreting human fibroblast feeder cells: a novel culture system for human embryonic stem cells. Mol Reprod Dev. 2008;75:1523-1532.

24. Li XF, Chen YL, Scheele S, et al. Fibroblast growth factor signaling and basement membrane assembly are connected during epithelial morphogenesis of the embryoid body. J Cell Biol. 2001;153:811-822.

25. Bendall SC, Stewart MH, Menendez P, et al. IGF and FGF cooperatively establish the regulatory stem cell niche of pluripotent human cells in vitro. Nature. 2007;448:1015-1021.

26. Garcia-Gonzalo FR, Izpisua Belmonte JC. Albumin-associated lipids regulate human embryonic stem cell self-renewal. PLoS One. 2008;3:e1384.

27. Rothschild MA, Oratz M, Schreiber SS. Regulation of albumin metabolism. Annu Rev Med. 1975;26:91-104.

28. Bendall SC, Hughes C, Campbell JL, et al. An enhanced mass spectrometry approach reveals human embryonic stem cell growth factors in culture. Mol Cell Proteomics. 2009;8:421-432.

29. Sarkar P, Collier TS, Randall SM, et al. The subcellular proteome of undifferentiated human embryonic stem cells. Proteomics. 2012;12:421430.

30. Sarkar P, Randall SM, Muddiman DC, et al. Targeted proteomics of the secretory pathway reveals the secretome of mouse embryonic fibroblasts and human embryonic stem cells. Mol Cell Proteomics. 2012;11:1829-1839.

31. Xiao L, Yuan X, Sharkis SJ. Activin A maintains self-renewal and regulates fibroblast growth factor, Wnt, and bone morphogenic protein pathways in human embryonic stem cells. Stem Cells. 2006;24:1476-1486.

32. Chen G, Gulbranson DR, Hou Z, et al. Chemically defined conditions for human iPSC derivation and culture. Nature methods. 2011;8:424-429.

33. Wang L, Schulz TC, Sherrer ES, et al. Self-renewal of human embryonic stem cells requires insulin-like growth factor-1 receptor and ERBB2 receptor signaling. Blood. 2007;110:4111-4119. 
34. Duntas LH. Selenium and inflammation: underlying anti-inflammatory mechanisms. Horm Metab Res. 2009;41:443-447.

35. Kim YH, Ryu JM, Lee YJ, et al. Fibronectin synthesis by high glucose level mediated proliferation of mouse embryonic stem cells: involvement of ANG II and TGF-beta1. J Cell Physiol. 2010;223:397-407.

36. Braam SR, Zeinstra $L$, Litjens $S$, et al. Recombinant vitronectin is a functionally defined substrate that supports human embryonic stem cell self-renewal via alphavbeta5 integrin. Stem Cells. 2008;26:2257-2265.

37. Vallier L, Reynolds D, Pedersen RA. Nodal inhibits differentiation of human embryonic stem cells along the neuroectodermal default pathway. Dev Biol. 2004;275:403-421.

38. Vallier L, Mendjan S, Brown S, et al. Activin/Nodal signalling maintains pluripotency by controlling Nanog expression. Development. 2009;136:1339-1349.

39. Vallier L, Alexander M, Pedersen RA. Activin/Nodal and FGF pathways cooperate to maintain pluripotency of human embryonic stem cells. J Cell Sci. 2005;118:4495-4509.

40. Kinehara $M$, Kawamura $S$, Tateyama $D$, et al. Protein kinase $C$ regulates human pluripotent stem cell self-renewal. PLoS One. 2013;8:e54122.

41. Ogawa $K$, Saito A, Matsui $H$, et al. Activin-Nodal signaling is involved in propagation of mouse embryonic stem cells. J Cell Sci. 2007;120:55-65.

42. Singh AM, Reynolds $D$, Cliff T, et al. Signaling network crosstalk in human pluripotent cells: a Smad2/3-regulated switch that controls the balance between self-renewal and differentiation. Cell Stem Cell. 2012;10:312-326.

43. Babaie Y, Herwig R, Greber B, et al. Analysis of Oct4-dependent transcriptional networks regulating self-renewal and pluripotency in human embryonic stem cells. Stem Cells. 2007:25:500-510.

44. Yamasaki S, Taguchi Y, Shimamoto A, et al. Generation of human induced pluripotent stem (Ips) cells in serum- and feeder-free defined culture and TGF-Beta1 regulation of pluripotency. PLoS One. 2014;9:e87151

45. Nakagawa M, Taniguchi $Y$, Senda $S$, et al. A novel efficient feeder-free culture system for the derivation of human induced pluripotent stem cells. Sci Rep. 2014;4:3594.

46. Gumbiner BM. Cell adhesion: the molecular basis of tissue architecture and morphogenesis. Cell. 1996;84:345-357.

47. Takeichi M. Cadherin cell adhesion receptors as a morphogenetic regulator. Science. 1991;251:1451-1455.

48. Geiger B, Ayalon O. Cadherins. Annu Rev Cell Biol. 1992;8:307-332.

49. Kemler R. From cadherins to catenins: cytoplasmic protein interactions and regulation of cell adhesion. Trends Genet. 1993;9:317-321.

50. Takeichi M. Morphogenetic roles of classic cadherins. Curr Opin Cell Biol. 1995;7:619-627.

51. Hatta K, Takeichi M. Expression of N-cadherin adhesion molecules associated with early morphogenetic events in chick development. Nature. 1986;320:447-449.

52. Nose A, Takeichi M. A novel cadherin cell adhesion molecule: its expression patterns associated with implantation and organogenesis of mouse embryos. J Cell Biol. 1986;103:2649-2658.

53. Cavallaro U, Christofori G. Cell adhesion and signalling by cadherins and Ig-CAMs in cancer. Nat Rev Cancer. 2004;4:118-132.

54. Gumbiner BM. Regulation of cadherin-mediated adhesion in morphogenesis. Nat Rev Mol Cell Bio. 2005;6:622-634.

55. Larue $L$, Antos $C$, Butz $S$, et al. A role for cadherins in tissue formation. Development. 1996;122:3185-3194.

56. Dang SM, Gerecht-Nir S, Chen J, et al. Controlled, scalable embryonic stem cell differentiation culture. Stem Cells. 2004;22:275-282.

57. Li L, Wang SA, Jezierski A, et al. A unique interplay between Rap1 and Ecadherin in the endocytic pathway regulates self-renewal of human embryonic stem cells. Stem Cells. 2010;28:247-257.

58. Ohgushi M, Sasai Y. Lonely death dance of human pluripotent stem cells: ROCKing between metastable cell states. Trends Cell Biol. 2011;21:274-282

59. De Santis G, Miotti S, Mazzi M, et al. E-cadherin directly contributes to $\mathrm{PI} 3 \mathrm{~K} / \mathrm{AKT}$ activation by engaging the PI3K-p85 regulatory subunit to adherens junctions of ovarian carcinoma cells. Oncogene. 2009;28:1206-1217.

60. Pece $S$, Chiariello M, Murga C, et al. Activation of the protein kinase Akt/ PKB by the formation of E-cadherin-mediated cell-cell junctions. Evidence for the association of phosphatidylinositol 3-kinase with the Ecadherin adhesion complex. J Biol Chem. 1999;274:19347-19351.

61. Goodwin M, Kovacs EM, Thoreson MA, et al. Minimal mutation of the cytoplasmic tail inhibits the ability of E-cadherin to activate Rac but not phosphatidylinositol 3-kinase: direct evidence of a role for cadherinactivated Rac signaling in adhesion and contact formation. J Biol Chem. 2003;278:20533-20539.

62. Thiery JP. Cell adhesion in development: a complex signaling network Curr Opin Genet Dev. 2003;13:365-371.

63. Nakagawa M, Fukata $M$, Yamaga $M$, et al. Recruitment and activation of Rac1 by the formation of E-cadherin-mediated cell-cell adhesion sites. J Cell Sci. 2001;114:1829-1838.

64. Fukata M, Kaibuchi K. Rho-family GTPases in cadherin-mediated cell-cell adhesion. Nat Rev Mol Cell Biol. 2001;2:887-897.

65 . Rizzino A. Stimulating progress in regenerative medicine: improving the cloning and recovery of cryopreserved human pluripotent stem cells with ROCK inhibitors. Regen Med. 2010;5:799-807.

66. Ohgushi $M$, Matsumura $M$, Eiraku $M$, et al. Molecular pathway and cell state responsible for dissociation-induced apoptosis in human pluripotent stem cells. Cell Stem Cell. 2010;7:225-239.

67. Soncin F, Ward CM. The function of e-cadherin in stem cell pluripotency and self-renewal. Genes (Basel). 2011;2:229-259.

68. Qian X, Anzovino A, Kim S, et al. N-cadherin/FGFR promotes metastasis through epithelial-to-mesenchymal transition and stem/progenitor celllike properties. Oncogene. 2014;33:3411-3421.

69. Stenzel D, Nye E, Nisancioglu $M$, et al. Peripheral mural cell recruitment requires cell-autonomous heparan sulfate. Blood. 2009;114:915-924.

70. Tran NL, Adams DG, Vaillancourt RR, et al. Signal transduction from $\mathrm{N}$-cadherin increases $\mathrm{Bcl}-2$. Regulation of the phosphatidylinositol 3-kinase/Akt pathway by homophilic adhesion and actin cytoskeletal organization. J Biol Chem. 2002;277:32905-32914.

71. Zhang JN, Shemezis JR, McQuinn ER, et al. AKT activation by N-cadherin regulates beta-catenin signaling and neuronal differentiation during cortical development. Neural Dev. 2013;8.

72. Bedzhov I, Alotaibi $\mathrm{H}$, Basilicata MF, et al. Adhesion, but not a specific cadherin code, is indispensable for ES cell and induced pluripotency. Stem Cell Res. 2013;11:1250-1263.

73. Vieira AF, Ribeiro AS, Dionisio MR, et al. P-cadherin signals through the laminin receptor alpha6beta4 integrin to induce stem cell and invasive properties in basal-like breast cancer cells. Oncotarget. 2014;5:679-692.

74. Shaw LM, Rabinovitz I, Wang HH, et al. Activation of phosphoinositide 3 $\mathrm{OH}$ kinase by the alpha6beta4 integrin promotes carcinoma invasion. Cell. 1997;91:949-960.

75. Bachelder RE, Ribick MJ, Marchetti A, et al. p53 inhibits alpha 6 beta 4 integrin survival signaling by promoting the caspase 3-dependent cleavage of AKT/PKB. J Cell Biol. 1999;147:1063-1072.

76. Marinkovich MP. Tumour microenvironment: laminin 332 in squamouscell carcinoma. Nat Rev Cancer. 2007;7:370-380.

77. Deugnier MA, Faraldo MM, Rousselle $P$, et al. Cell-extracellular matrix interactions and EGF are important regulators of the basal mammary epithelial cell phenotype. J Cell Sci. 1999;112:1035-1044.

78. Cheung LW, Leung PC, Wong AS. Cadherin switching and activation of p120 catenin signaling are mediators of gonadotropin-releasing hormone to promote tumor cell migration and invasion in ovarian cancer. Oncogene. 2010;29:2427-2440.

79. Giancotti FG, Ruoslahti E. Integrin signaling. Science. 1999;285:1028-1032.

80. Brafman DA, Phung C, Kumar N, et al. Regulation of endodermal differentiation of human embryonic stem cells through integrin-ECM interactions. Cell Death Differ. 2013;20:369-381.

81. Evseenko D, Schenke-Layland K, Dravid G, et al. Identification of the critical extracellular matrix proteins that promote human embryonic stem cell assembly. Stem Cells Dev. 2009;18:919-928.

82. Hynes RO. Integrins: bidirectional, allosteric signaling machines. Cell. 2002;110:673-687.

83. Chang BS, Choi YJ, Kim JH. Collagen complexes increase the efficiency of iPS cells generated using fibroblasts from adult mice. J Reprod Dev. 2015;61:145-153.

84. Li HY, Liao CY, Lee KH, et al. Collagen IV significantly enhances migration and transplantation of embryonic stem cells: involvement of alpha2beta1 integrin-mediated actin remodeling. Cell Transplant. 2011;20:893-907.

85. Grzesiak JJ, Bouvet M. The alpha2beta1 integrin mediates the malignan phenotype on type I collagen in pancreatic cancer cell lines. Br J Cancer 2006;94:1311-1319.

86. Diez-Torre A, Silvan U, De Wever O, et al. Germinal tumor invasion and the role of the testicular stroma. Int J Dev Biol. 2004;48:545-557. 
87. Yamana R, Iwasaki M, Wakabayashi M, et al. Rapid and deep profiling of human induced pluripotent stem cell proteome by one-shot NanoLCMS/MS analysis with meter-scale monolithic silica columns. J Proteome Res. 2013;12:214-221.

88. Kanematsu A, Marui A, Yamamoto S, et al. Type I collagen can function as a reservoir of basic fibroblast growth factor. J Control Release. 2004;99:281-292.

89. Suh HN, Han HJ. Collagen I regulates the self-renewal of mouse embryonic stem cells through alpha2beta1 integrin- and DDR1-dependent Bmi-1. J Cell Physiol. 2011;226:3422-3432.

90. Rodin S, Domogatskaya A, Strom S, et al. Long-term self-renewal of human pluripotent stem cells on human recombinant laminin-511. Nat Biotechnol. 2010;28:611-615.

91. Gu J, Fujibayashi A, Yamada KM, et al. Laminin-10/11 and fibronectin differentially prevent apoptosis induced by serum removal via phosphatidylinositol 3-kinase/Akt- and MEK1/ERK-dependent pathways. J Biol Chem. 2002;277:19922-19928.

92. Domogatskaya A, Rodin S, Boutaud A, et al. Laminin-511 but not -332, -111 , or -411 enables mouse embryonic stem cell self-renewal in vitro. Stem Cells. 2008;26:2800-2809.

93. Semba K, Nishizawa M, Miyajima N, et al. yes-related protooncogene, syn, belongs to the protein-tyrosine kinase family. Proc Natl Acad Sci USA. 1986;83:5459-5463.

94. Baer AS, Syed YA, Kang SU, et al. Myelin-mediated inhibition of oligodendrocyte precursor differentiation can be overcome by pharmacological modulation of Fyn-RhoA and protein kinase $C$ signalling. Brain. 2009;132:465-481.

95. Liang X, Draghi NA, Resh MD. Signaling from integrins to Fyn to Rho family GTPases regulates morphologic differentiation of oligodendrocytes. J Neurosci. 2004;24:7140-7149.

96. Colognato $\mathrm{H}$, Ramachandrappa $\mathrm{S}$, Olsen IM, et al. Integrins direct Src family kinases to regulate distinct phases of oligodendrocyte development. J Cell Biol. 2004;167:365-375.

97. Sroka IC, Anderson TA, McDaniel KM, et al. The laminin binding integrin alpha6beta1 in prostate cancer perineural invasion. J Cell Physiol. 2010;224:283-288.

98. Litjens SH, de Pereda JM, Sonnenberg A. Current insights into the formation and breakdown of hemidesmosomes. Trends Cell Biol. 2006;16:376-383.

99. Klein S, Giancotti FG, Presta M, et al. Basic fibroblast growth factor modulates integrin expression in microvascular endothelial cells. Mol Biol Cell. 1993;4:973-982.

100. Ruoslahti E, Pierschbacher MD. New Perspectives in Cell-Adhesion - Rgd and Integrins. Science. 1987;238:491-497.

101. Humphries JD, Byron A, Humphries MJ. Integrin ligands at a glance. J Cell Sci. 2006;119:3901-3903.

102. Ruoslahti E. RGD and other recognition sequences for integrins. Ann Rev Cell Dev Biol. 1996;12:697-715.

103. Danen $E H$, Sonneveld $P$, Brakebusch $C$, et al. The fibronectin-binding integrins alpha5beta1 and alphavbeta3 differentially modulate RhoAGTP loading, organization of cell matrix adhesions, and fibronectin fibrillogenesis. J Cell Biol. 2002;159:1071-1086.

104. Amit M, Shariki C, Margulets V, et al. Feeder layer- and serum-free culture of human embryonic stem cells. Biol Reprod. 2004;70:837-845.

105. Huveneers S, Truong $H$, Fassler R, et al. Binding of soluble fibronectin to integrin alpha5 beta1-link to focal adhesion redistribution and contractile shape. J Cell Sci. 2008;121:2452-2462.

106. Lee JW, Juliano RL. The alpha5beta1 integrin selectively enhances epidermal growth factor signaling to the phosphatidylinositol-3-kinase/Akt pathway in intestinal epithelial cells. Biochim Biophys Acta. 2002;1542:23-31.

107. Chang YQ, Li H, Guo ZK. Mesenchymal Stem Cell-Like Properties in Fibroblasts. Cell Physiol Biochem. 2014;34:703-714.

108. Farias E, Lu M, Li X, et al. Integrin alpha8beta1-fibronectin interactions promote cell survival via PI3 kinase pathway. Biochem Biophys Res Commun. 2005;329:305-311.

109. Vogel BE, Tarone G, Giancotti FG, et al. A novel fibronectin receptor with an unexpected subunit composition (alpha v beta 1). J Biol Chem. 1990;265:5934-5937.

110. Bodary SC, Mclean JW. The integrin beta-1 subunit associates with the vitronectin receptor alpha- $\mathrm{V}$ subunit to form a novel vitronectin receptor in a human embryonic kidney-cell line. J Biol Chem. 1990;265:5938-5941.
111. Aplin JD, Sattar A, Mould AP. Variant choriocarcinoma (BeWo) cells that differ in adhesion and migration on fibronectin display conserved patterns of integrin expression. J Cell Sci. 1992;103:435-444.

112. Dedhar S, Gray V. Isolation of a novel integrin receptor mediating ArgGly-Asp directed cell-adhesion to fibronectin and type-I collagen from human neuroblastoma-cells - association of a novel beta-1-related subunit with alpha-V. J Cell Biol. 1990;110:2185-2193.

113. Marshall JF, Rutherford DC, Mccartney ACE, et al. Alpha(V)Beta(1) is a receptor for vitronectin and fibrinogen, and acts with alpha(5)beta(1) to mediate spreading on fibronectin. J Cell Sci. 1995;108:1227-1238.

114. Munger JS, Huang $X$, Kawakatsu $H$, et al. The integrin alpha $v$ beta 6 binds and activates latent TGF beta 1: a mechanism for regulating pulmonary inflammation and fibrosis. Cell. 1999;96:319-328.

115. Prowse AB, Doran MR, Cooper-White JJ, et al. Long term culture of human embryonic stem cells on recombinant vitronectin in ascorbate free media. Biomaterials. 2010;31:8281-8288.

116. Aluwihare $P, M u Z Y, Z$ hao $Z C$, et al. Mice that lack activity of alpha $v$ beta 6-and alpha $v$ beta 8-integrins reproduce the abnormalities of Tgfb1and Tgfb3-null mice. J Cell Sci. 2009;122:227-232.

117. Yang ZW, Mu ZY, Dabovic B, et al. Absence of integrin-mediated TGF beta 1 activation in vivo recapitulates the phenotype of TGF beta 1-null mice. J Cell Biol. 2007;176:787-793.

Cite this article as: Nakashima Y, Omasa T (2016) What kind of sig naling maintains pluripotency and viability in human induced pluripotent stem cells cultured on laminin-511 with serum-free medium? BioResearch Open Access 5:1, 84-93, DOI: 10.1089/biores.2016.0001.

\begin{aligned} \multicolumn{1}{c|}{ Abbreviations Used } \\ $\mathrm{ALK}=$ activin-like kinase \\ $\mathrm{BSA}=$ bovine serum albumin \\ $\mathrm{CAMs}=$ cell adhesion molecules \\ $\mathrm{ECM}=$ extracellular matrix \\ $\mathrm{ERK}=$ extracellular signal-regulated kinase \\ $\mathrm{FGFR}=$ fibroblast growth factor receptor \\ $\mathrm{HSPG}=$ heparan sulfate proteoglycans \\ $\mathrm{hiPSC}=$ human-induced pluripotent stem cells \\ $\mathrm{hPSC}=$ human pluripotent stem cell \\ $\mathrm{IGF}=$ insulin-like growth factor \\ $\mathrm{ITS}=$ insulin, transferrin, and selenium \\ $\mathrm{MAPK}=$ mitogen-activated protein kinase \\ $\mathrm{MEF}=$ mouse embryonic fibroblast \\ $\mathrm{NF}-\kappa \mathrm{B}=$ nuclear factor $\kappa \mathrm{B} \\ \mathrm{TGF}-\beta=$ transforming growth factor $\beta\end{aligned}$

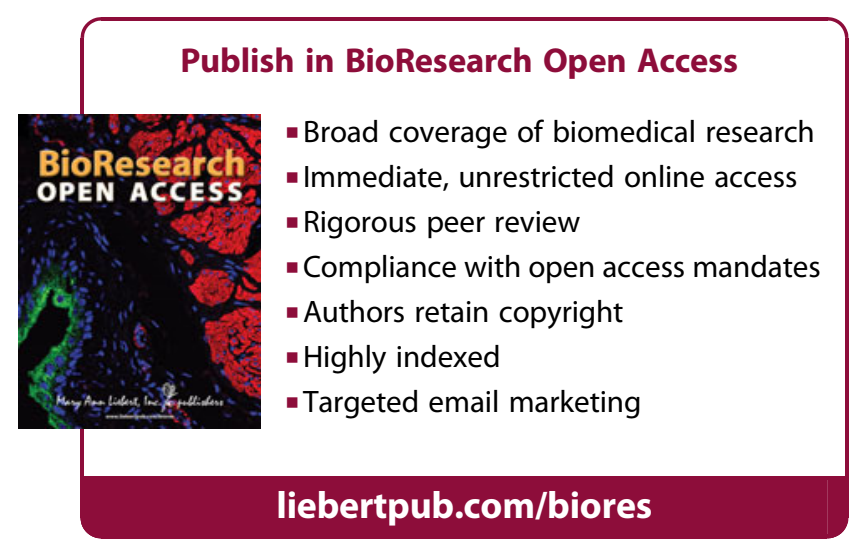

